\title{
TECHNICAL AND ECONOMIC FEASIBILITY OF REUSING DISPOSABLE PERFUSION CANNULAS
}

David F. Bloom, MS ${ }^{\mathrm{a}}$

J. Fredrick Cornhill, DPhil ${ }^{\mathrm{a}}$

Paul S. Malchesky, DEng ${ }^{\mathrm{c}}$

Donna M. Richardson, $\mathrm{MS}^{\mathrm{c}}$

Kathryn A. Bolsen, BS

Donna M. Haire, BS ${ }^{\circ}$

Floyd D. Loop, MD $^{\mathrm{b}}$

Delos M. Cosgrove III, MD
Objective(s): The reuse of disposable devices is a potential source of significant cost savings to hospitals. Venous and arterial perfusion cannulas under new and reused conditions were selected to identify the clinical, safety, technical, logistic, and economic issues that must be addressed to realize these savings. Methods: Single- and dual-stage venous and arterial cannulas from two manufacturers were tested when new, after initial clinical use, and after a single clinical use plus up to nine simulated reuses. Reuse was simulated by end-to-end bending, coupling and uncoupling of the connectors, and by two 1-hour soaks in plasma at $4^{\circ}$ and $40^{\circ} \mathrm{C}$, respectively. Cannulas were decontaminated and then processed by a peracetic acid-based liquid chemical sterilization system after each use/reuse. Sterilization was validated by eliminating Bacillus subtilis spores from the cannulas on each of five consecutive cycles. Cannulas were tested for physical changes, functional integrity, biocompatibility, and in vivo performance in sheep. A cost analysis was also performed. Results: Sterilization was successfully achieved. Mechanical changes were less than $20 \%$ on all variables studied and were undetectable by experienced cardiac surgeons in selective evaluation. No clinically important differences were found between new and reused cannulas, even after nine simulated reuses. Reusing cannulas four times would reduce the cost per procedure from $\$ 53$ to $\$ 19(64 \%)$. Conclusions: Preliminary data suggest that the perfusion cannulas tested can be safely and efficaciously used five times. Limited reuse of these disposable cannulas is technically feasible and costeffective. Cannula reuse would result in a small incremental savings; however, with more expensive devices and higher-volume sterilization procedures, the savings could be considerably greater. This program provides a model for evaluation of other single-use medical devices for reuse. (J Thorac Cardiovasc Surg 1997;114:448-60)
$T^{1}$ he cost of disposable products has contributed markedly to the expense of surgical procedures. ${ }^{1}$

Limited reimbursement for procedures coupled with institutional cost-containment of health care provision has caused attention to be focused on the

From the Departments of Biomedical Engineering ${ }^{\mathrm{a}}$ and Thoracic and Cardiovascular Surgery, ${ }^{\mathrm{b}}$ The Cleveland Clinic Foundation, Cleveland, Ohio, and STERIS Corporation, ${ }^{c}$ Mentor, Ohio.

This study was presented in part at the AAMI/FDA conference entitled "Designing, Testing, and Labeling Reusable Medical Devices for Reprocessing in Health Care Facilities," November 13-15, 1996, Los Angeles, and a poster appears in Biomedical Instrumentation and Technology, May/June 1997.

Received for publication Nov. 13, 1996; accepted for publication Feb. 21, 1997.

Address for reprints: J. Fredrick Cornhill, DPhil, Chairman, Department of Biomedical Engineering, The Cleveland Clinic Foundation, 9500 Euclid Ave., Cleveland, OH 44195.

Copyright $@ 1997$ by Mosby-Year Book, Inc.

$0022-5223 / 97 \$ 5.00+0 \quad \mathbf{1 2 / 1 / 8 1 3 9 2}$ expenditures for disposable medical products and the possible cost savings if such products could be reused. To investigate the feasibility of limited reuse of disposable products, we assessed our institution's use of disposable devices. Particular attention was given to high-cost and low-cost/high-volume products in which a marked cost savings might be realized. Once candidate single-use products were identified for study, a program was developed to evaluate the safety and efficacy of reprocessing these devices.

Focus was drawn quickly to the reuse of perfusion cannulas because cannulas are used in large numbers, are of simple design, and have a history of reuse in our institution. Cannula reuse was a widely accepted practice in the early years of bypass surgery. However, when manufacturers increased the availability of single-use disposable cannulas, reuse was discontinued. Another reason for concentrating on perfusion cannulas is that the prerequisites for 
reuse of these devices, including sterilization efficacy, materials compatibility, biocompatibility, and device functionability, are similar to safety criteria for other single-use products.

\section{Materials and methods}

Cannula types. Venous and arterial cannulas with the highest volume of use in this institution were chosen for study. These were the Research Medical Incorporated (RMI, Salt Lake City, Utah) dual- and single-stage venous return cannulas $(32 \mathrm{~F}$ and $36 \mathrm{~F}$ ) and the $3 \mathrm{M} /$ Sarns (St. Paul, Minn.) Soft Flow $8.0 \mathrm{~mm}$ arterial cannula models.

Cannula groups. Cannulas were divided into three main groups for testing: (1) new (unused), (2) one clinical use, and (3) one clinical use and nine simulated uses. Unused cannulas (taken directly from the manufacturer's original packaging) were evaluated to establish a baseline level. The clinically used cannulas were cleaned, decontaminated, and put through a sterilization cycle after the initial clinical use and each subsequent simulated (re)use. It was estimated that five clinical uses would offer considerable cost savings for these devices. Therefore a total of ten uses/reuses was chosen as an end point to provide a margin of safety by doubling the cycle requirements. Some testing also evaluated the cannulas after five simulated reuses.

Cannula cleaning and decontamination. After clinical use, the cannulas were retrieved from the operating room, cleaned, and decontaminated in the Surgical Processing Department of The Cleveland Clinic Foundation with an institutionally approved cleaning and decontamination protocol. Cleaning and decontamination consisted of the following steps: (1) rinsing the cannulas with tap water, (2) soaking, flushing, and sonicating the cannulas in a warm enzymatic detergent solution (Enzol, Johnson \& Johnson Medical Inc., Arlington, Tex.), (3) rinsing them with deionized water, and (4) drying them with compressed air. Each cannula was then assigned an identification number and put through a sterilization cycle.

Simulated use. Use of the cannulas was simulated by flexing them end to end up to ten times, connecting the proximal end to the blood tubing adaptor (for RMI cannulas) or blood tubing (for $3 \mathrm{M} / \mathrm{Sarns}$ cannulas), and attaching the appropriate connector tie. In addition, the cannulas were placed in human plasma and refrigerated for 1 hour at $4^{\circ} \mathrm{C}$, which is the lowest temperature maintained during bypass surgery, for that length of time. The cannulas then were soaked in human plasma for an additional. hour at about $40^{\circ} \mathrm{C}$, the highest temperature reached during surgery. After the plasma soak periods, the cannulas were rinsed with saline solution, cleaned, decontaminated, and put through a sterilization cycle.

Sterilization processing. The cannulas were processed in the STERIS SYSTEM 1 Processor (STERIS Corporation, Mentor, Ohio). This system has been cleared by the Food and Drug Administration (FDA) for the sterilization of reusable immersible medical, surgical, and diagnostic devices. The SYSTEM 1 Processor, which is available at the site of care, is a table-top microprocessorcontrolled chamber in which the liquid chemical sterilization process is performed, controlled, and monitored.
STERIS 20 Sterilant Concentrate was used as the chemical germicide for sterilization. STERIS 20 is a single-use sterilant registered by the U.S. Environmental Protection Agency, which has been developed specifically for use with the STERIS PROCESS. It contains the active sporicidal ingredient peracetic acid with a proprietary chemical buffering, anticorrosive formulation. STERIS 20 is mixed automatically with water that has been filtersterilized by the processor to form a solution. This solution flows and mixes in the processor's chamber, thereby coming into contact with all accessible external and internal surfaces of the cannulas. The sterilization phase of the process lasts 12 minutes and is followed by four sterile water rinses to free the devices of sterilant residues. The entire process is completed in about 30 minutes.

Up to four cannulas per cycle were processed in the STERIS General Processing Container. The cannulas were placed on a rack in the STERIS General Processing Container with the large lumen end elevated.

Validation of sterilization. Individual cannulas were inoculated with a Bacillus subtilis (ATCC No. 19659) spore suspension of at least $10^{7}$ colony-forming units (cfu) per milliliter by means of a syringe filled with this spore solution. Bacillus subtilis spores were chosen as the test organisms because they are highly resistant to the sterilant. Harvesting was performed after processing by swabbing selected sites on the body, ends, and lumen of the cannulas. After swabbing, the lumen was eluted with $10 \mathrm{ml}$ of sterile water, which was collected in a test tube containing $10 \mathrm{ml}$ double-strength tryptic soy broth; the resulting mixture was cultured. The swab and elution samples in tryptic soy broth were cultured for 7 days at $37^{\circ} \mathrm{C}$. For sterilization to be considered valid, the absence of culture growth in all samples was required for five consecutive sterilization cycles. A recoverable bioburden evaluation was also performed to determine the level of inoculum adhering to the cannulas. Recoverable bioburden samples were quantified by plating them on tryptic soy agar for 48 hours at $37^{\circ} \mathrm{C}$. In addition, a positive control cycle (in which the sterilant's active ingredient, peracetic acid, is omitted) was run to demonstrate that the acid is required for sporicidal activity. This was demonstrated by evaluating the presence of spores adhering to the cannula test sites by means of swabbing and elution techniques after the control cycle.

Materials and function testing. The effects of reprocessing on the functional properties and characteristics of the cannulas were tested. Materials were evaluated by assessing their physical appearance, color, tactile properties, hardness, and dimensional changes after simulated use and sterilization. Two function tests were also performed: (1) a cantilever bend test $^{2}$ (which measures cannula displacement as a function of a hanging weight) to assess the flexibility and bending strength of the cannula's body and tip and (2) a tensile test ${ }^{3}$ to assess the integrity of the joints and bonds along its length. Because the distal tip of the $3 \mathrm{M} / \mathrm{Sarns}$ arterial cannula is connected to the body by struts, these cannulas were evaluated during mechanical shear conditions as well. All tensile and shear tests were performed on a Bionix Test System (model 858; MTS Systems Corp., Minneapolis, Minn.) 


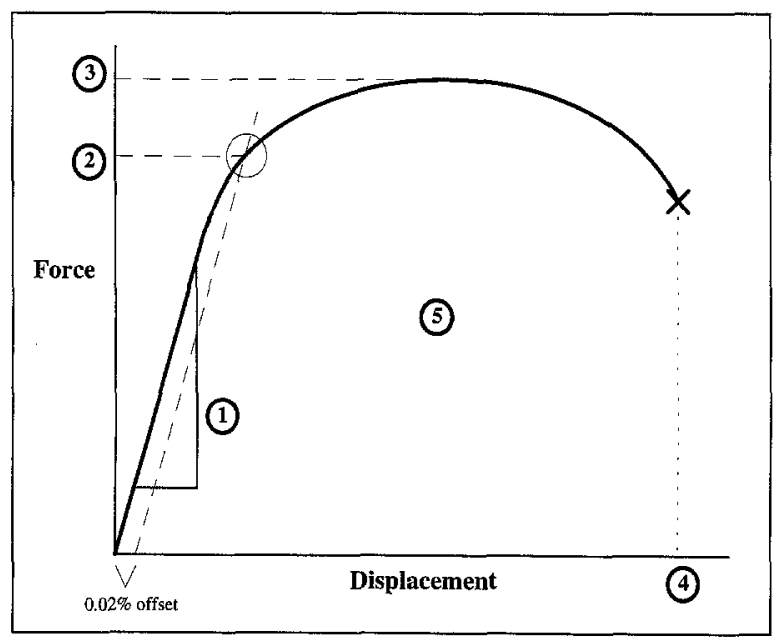

Fig. 1. Mechanical properties obtained from tensile tests. The diagram is a representative force-displacement graph obtained from a tensile test. The diagram illustrates the mechanical properties determined from the force-displacement curve. The mechanical properties are as follows: 1 , Tensile stiffness: The slope of the linear (elastic) portion of the force-displacement curve. 2, Yield strength: The point along the force-displacement curve that designates the transition from elastic (recoverable) to plastic (permanent) deformation by means of the $0.02 \%$ strain offset method. The intersection of the force-displacement curve and the dashed line (the offset line) designates the transition point. 3, Ultimate tensile strength: The maximum load/force experienced during the tensile test. 4, Elongation-at-break: The length to which the specimen is elongated at the point of failure. 5, Energy-to-break: The total area under the force-displacement curve.

with a Test Star Controller (model 490) and Test Ware-SX Applications Software (MTS Systems).

Tensile testing of the cannulas resulted in force-displacement curves (Fig. 1) from which five mechanical properties were measured: tensile stiffness, yield strength, ultimate tensile strength, elongation-at-break, and energyto-break. Tensile stiffness is the ratio of the applied force to the displacement and is a measure of the material's resistance to elastic (recoverable) deformation. Tensile stiffness was the only property that could be measured when testing was limited to below the device's yield point. Energy-to-break is the amount of energy (area under the force-displacement curve) required to cause a material to fail or break. Analogous properties were also obtained from the torque-displacement curves generated from the mechanical shear tests. The cantilever bend test displacement data and the mechanical properties described were analyzed by means of unpaired Student's $t$ tests to compare the new and used cannulas. All tests were two tailed and $\alpha$ was set at 0.05 .

Biocompatibility testing. Various tests were conducted to assess the biocompatibility of the cannulas. These tests included residue analysis (both cannula and sterilant), cytotoxicity, hemolysis, muscle implant, pyrogen/endotoxin analysis, and toxicity (systemic and intracutaneous). All of the testing except for the sterilant residue analysis was performed by an outside independent laboratory (NAmSA, Northwood, Ohio). Biocompatibility testing was performed on both RMI dual-stage venous and $3 \mathrm{M} /$ Sarns arterial perfusion cannulas. Unless otherwise noted, all of the test cannulas were clinically used once and then underwent nine simulated uses including eleven or twelve sterilization cycles. The RMI single-stage venous cannula model was not evaluated because its materials are identical to those of the dual-stage cannula.

To evaluate cannula extractables, extractions with U.S. Pharmacopeia (USP) purified water at $37^{\circ} \mathrm{C}$ for 24 hours were carried out on unused cannulas that were processed once and then compared with new cannula residue extracts. Each extract was dried at $100^{\circ} \mathrm{C}$ for 24 hours. A portion of the residue was dissolved in tetrahydrofuran. The solution was applied to a potassium bromide disc and the solvent was allowed to evaporate. Infrared analysis was performed on the resulting film to assess organic extractions.

The two cannula models were also extracted separately with $0.1 \mathrm{~N}$ sodium hydroxide and $0.1 \mathrm{~N}$ hydrochloric acid so that select sterilant components could be analyzed by two methods: (1) inductively coupled plasma emission spectrophotometry for elemental analyses and (2) ultraviolet spectrophotometry for an organic anticorrosive formulation agent. New, unused cannulas were used as controls. After the final sterilization cycle, test strips for hydrogen peroxide (EM Quant peroxide test strips, EM Science, Gibbstown, N.J.) and peracetic acid (Merckoquant peracetic acid strips, EM Science) were used to detect residues of these chemicals from the sterilization process.

Cytotoxicity testing of unused RMI dual-stage venous cannulas (processed once) and clinically used 3M/Sarns arterial cannulas (nine simulated uses and processed 12 times) was performed by means of the minimum essential medium elution method. The cannulas were extracted with $5 \%$ minimum esential medium solution at $37^{\circ} \mathrm{C}$ for 24 hours. A monolayer of L-929 mouse fibroblast cells (grown to confluency) was exposed to the extract, and after incubation the cells were evaluated for changes in cell shape.

In vitro hemolysis testing consisted of extracting the cannulas with a $0.9 \%$ sodium chloride solution at $70^{\circ} \mathrm{C}$ for 24 hours. Rabbit blood collected in ethylenediaminetetraacetic acid was added to a portion of the extract solution at room temperature. This solution was then heated to $37^{\circ} \mathrm{C}$ in a constant temperature bath for 1 hour and the percent of hemolysis was determined spectrophotometrically.

In the USP muscle implantation test, slivers of the test cannulas were implanted into the paravertebral muscle of New Zealand White albino rabbits for 7 days. After excision, the tissue surrounding each implant was macroscopically evaluated for evidence of encapsulation or adverse reaction. The implant sites were also histologically processed and microscopically evaluated.

Pyrogenicity testing involved assessing the presence of endotoxin using the Limulus amebocyte lysate gel test. For the USP systemic extract toxicity studied in mice and 


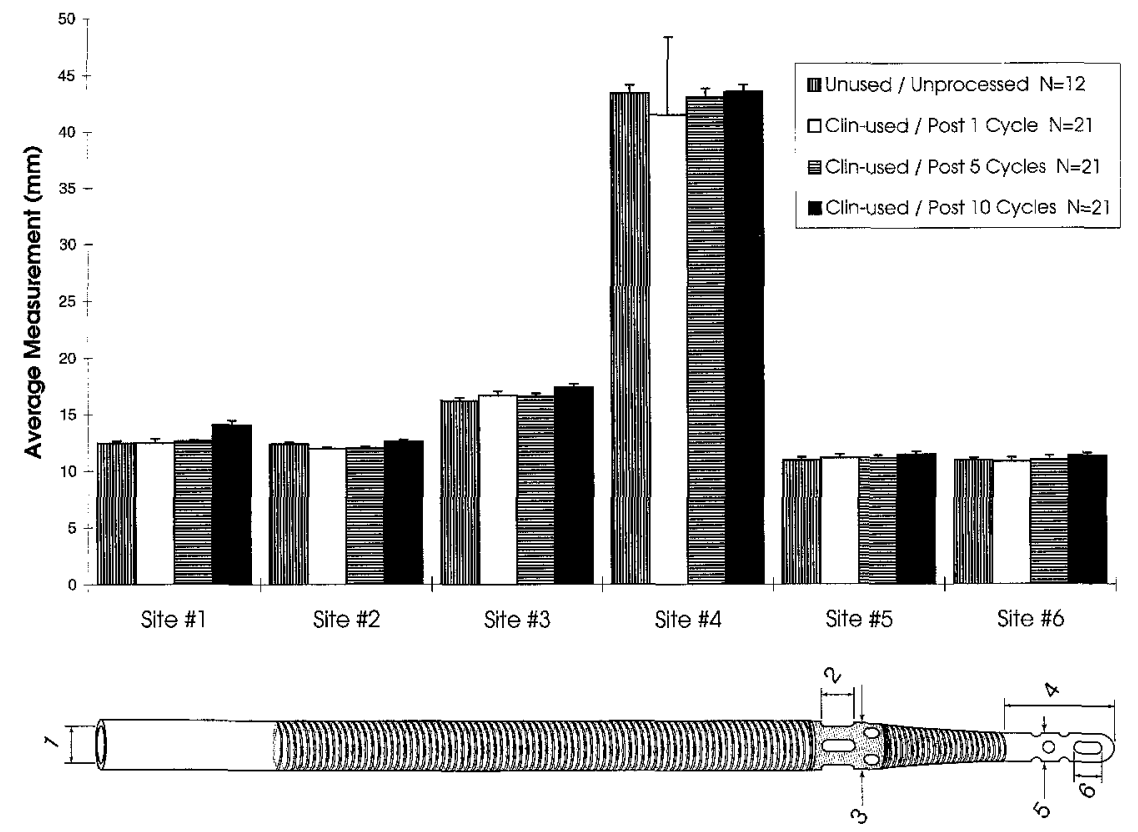

Fig. 2. Materials evaluation-Dimensions, RMI dual-stage venous cannula.

Table I. Sterilization validation data for cannulas challenged with Bacilus subtilis spore suspensions

\begin{tabular}{|c|c|c|c|}
\hline Cannula model & $\begin{array}{c}\text { Sterilization cycle } \\
\text { (No. sites sterile/No. tested) }\end{array}$ & $\begin{array}{c}\text { Positive control cycle } \\
\text { (No. sites not sterile/No. tested) }\end{array}$ & $\begin{array}{l}\text { Recoverable bioburden } \\
\text { (range: } \times 10^{3} \text { cfuldevice) }\end{array}$ \\
\hline \multicolumn{4}{|l|}{ RMI duall stage } \\
\hline Clinical use ${ }^{*}(n=5)$ & $100 / 100$ & $20 / 20$ & 3.84 to $\geq 68.1$ \\
\hline Simulated use $\dagger(n=5)$ & $100 / 100$ & $20 / 20$ & 31.8 to $\geq 84.3$ \\
\hline \multicolumn{4}{|l|}{ RMI single stage } \\
\hline Clinical use ${ }^{*}(n=5)$ & $75 / 75$ & $15 / 15$ & 7.17 to 15.3 \\
\hline Simulated use $\dagger(n=5)$ & $75 / 75$ & $15 / 15$ & 12.3 to $\geq 41.8$ \\
\hline \multicolumn{4}{|l|}{ 3M/Sarns } \\
\hline Clinical use* $(n=5)$ & $125 / 125$ & $25 / 25$ & 11.2 to $\geq 42.0$ \\
\hline Simulated use $\dagger(n=5)$ & $125 / 125$ & $25 / 25$ & 6.90 to 16.8 \\
\hline Total $(n=30)$ & $600 / 600$ & $120 / 120$ & $\begin{array}{c}\text { Overail range of } \\
\text { recoverable bioburden: } \\
3.84 \times 10^{3} \text { to } \geq 8.43 \times 10^{4} \mathrm{cfu} / \text { device }\end{array}$ \\
\hline
\end{tabular}

"Cannulas with one clinical use (one sterilization cycle).

$\dagger$ Cannulas with one clinical use and nine simulated uses (10 sterilization cycies).

the USP intracutaneous extract toxicity studied in rabbits, extracts of both saline solution and cottonseed oil from each of the two cannula models were studied.

In vivo testing. To assess the overall effect of multiple reprocessing and to allow for a qualitative evaluation by a surgeon, we analyzed RMI single-stage venous perfusion cannulas during surgical use. Two new cannulas and three used cannulas (clinically used once and processed four times without simulated use $=$ five total sterilization cycles) were used in sheep undergoing cardiac valve replacement as part of an animal research protocol. All animals used in this study received humane care in compliance with the "Principles of Laboratory Animal Care" formulated by the National Society for Medical
Research and the "Guide for the Care and Use of Laboratory Animals" prepared by the Institute of Laboratory Animal Resources and published by the National Institutes of Health (NIH Publication No. 86-23, revised 1985).

\section{Results}

Validity of sterilization. All clinically used and simulated-use cannulas that were inoculated with Bacillus subtilis spores showed no signs of the test organism after sterilization (Table I). In the positive control evaluation of the sterilization system without 


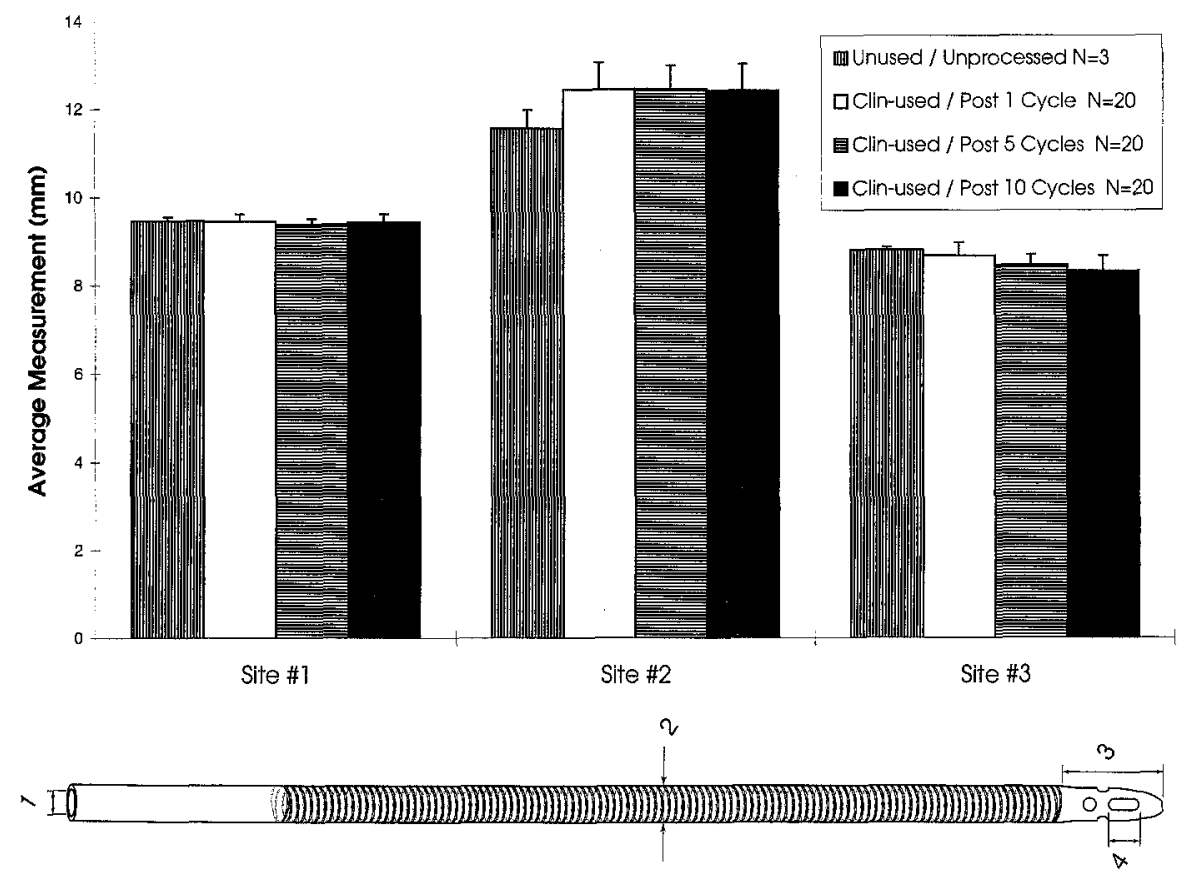

Fig. 3. Materials evaluation-Dimensions, RMI single-stage venous cannula.

the sporicidal agent peracetic acid, all sites showed growth. This result demonstrates that neither the fluid action during the cycle, the sterilant components (other than the peracetic acid), nor the successive sterile water rinses were responsible for the sporicidal activity.

In studies assessing the inoculated sites, recovery of Bacillus subtilis spores ranged from $3.84 \times 10^{3}$ to $8.43 \times 10^{4} \mathrm{cfu}$ or more per device (Table I). However, inasmuch as only discrete sites on the cannulas were recovered for bioburden, as opposed to evaluating the entire surface of the cannula, the recovery results underestimate the total level of inoculation.

After clinical use but before cleaning or sterilization, clinically used cannulas $(n=10)$ were evaluated for contamination. Growth was found on only two of the cannulas. The vegetative bacteria identified (presumptive Staphylococcus epidermidis) on these cannulas are significantly less resistant to the sterilant than the Bacillus subtilis spores used in this study. Furthermore, the concentration of the vegetative bacteria was very low ( $<20$ cfu per device) compared with the spore contamination levels on the cannulas measured in the recoverable bioburden evaluations.
Materials and function testing. After reprocessing, the appearance of the reused cannulas was not noticeably different from that of new, unused cannulas. Slight yellowing in the RMI dual-stage cannulas and in the $3 \mathrm{M} / \mathrm{Sarns}$ cannulas in the simulated-use group was noticed. The discoloration, which was a change in the material tint and not its opacity, has no known effect on the clinical properties of the cannulas. Localized darker yellowing was noted in some of the cannulas and appeared to be associated with imperfections in the material; this might be related to variability in the manufacturing process. No changes in texture occurred after reprocessing.

Variable amounts of surface scoring at the proximal end of the cannulas, where the plastic connectors are inserted, were noted on all RMI cannulas. This physical damage, which increased with the number of (re)uses, was related to the connector design and could be eliminated with more careful handling of the cannulas during insertion and removal of the connector or by using a connector with a smoother hose barb profile. Scoring did not occur on the $3 \mathrm{M} /$ Sarns cannulas because the connector is bonded to the cannula body and no assembly is required between the connector and cannula. 


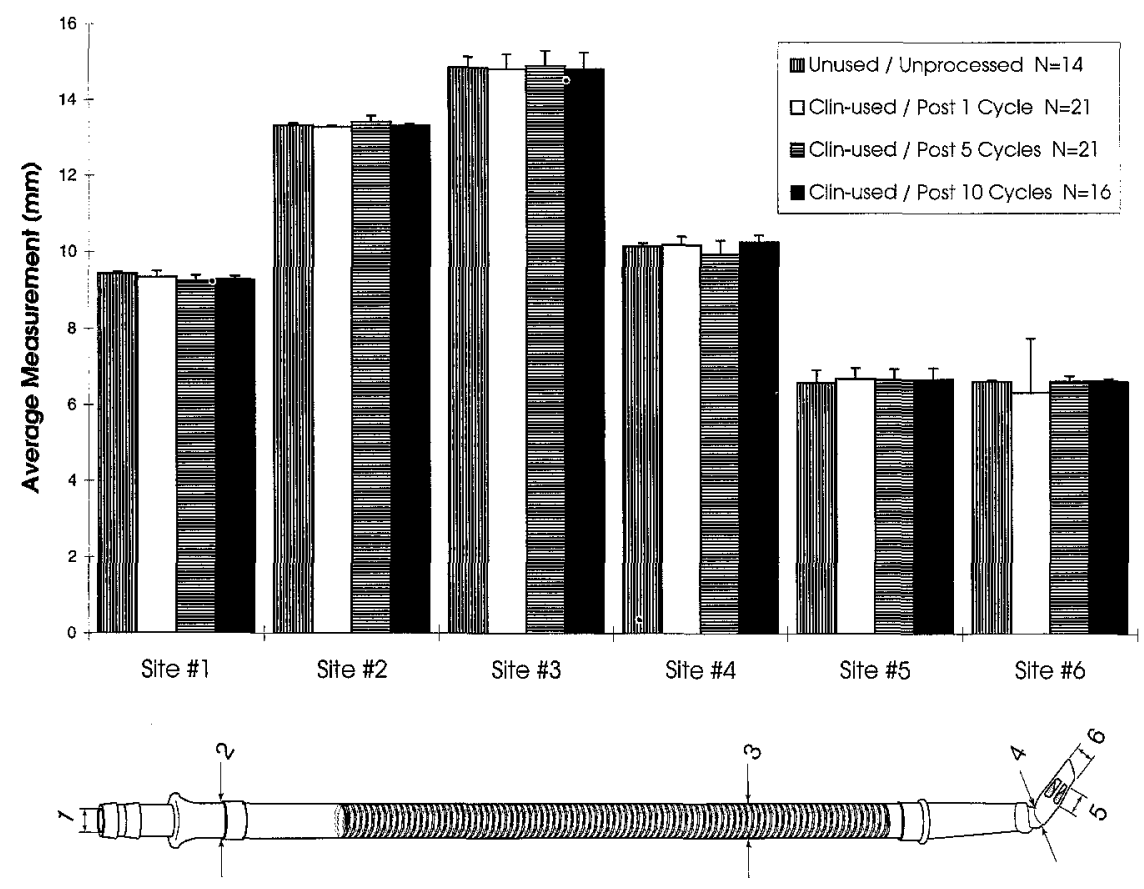

Fig. 4. Materials evaluation-Dimensions, $3 \mathrm{M} /$ Sarns arterial cannula.

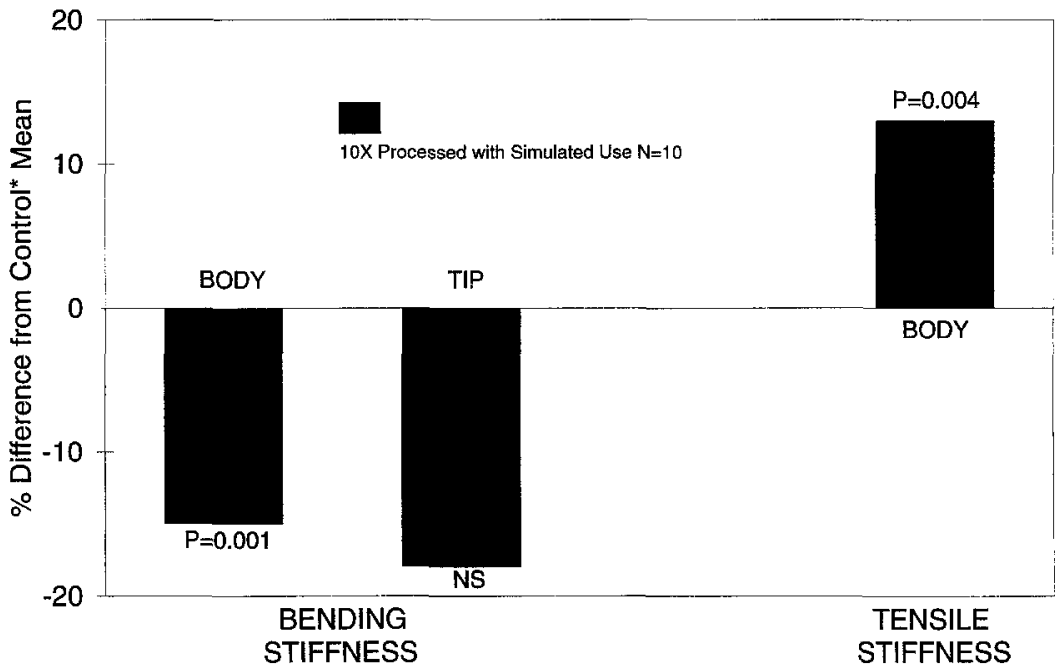

Fig. 5. Function testing $-22^{\circ} \mathrm{C}$, RMI dual-stage venous cannula. $N S$, Not significant. *Controls (new cannulas), $n=5$.

Slight dimensional changes were found at some sites on the three cannula models studied (Figs. 2 to 4). However, evaluations by a cardiac surgeon indicated that none of the changes were clinically important. For example, the internal diameter of the connector end on the RMI cannula models (which requires the insertion of a connector) did not change markedly. Thus the cannula's ability to be properly connected to the blood tubing and provide a leak-free conduit for blood flow was not impaired. Furthermore, durometer (hardness) assessments of the cannulas showed no statistically significant changes.

A statistically significant decrease in bending stiffness (resulting in an increase in displacement) was 


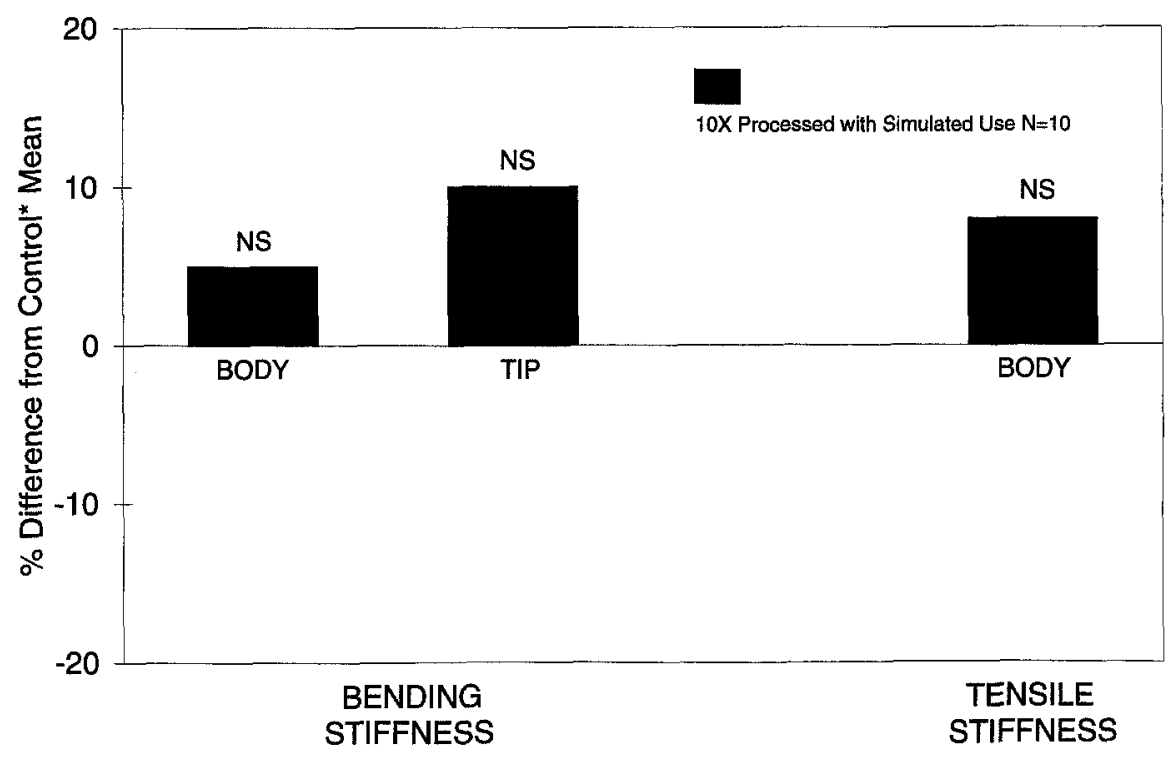

Fig. 6. Function testing- $22^{\circ} \mathrm{C}$, RMI single-stage venous cannula. $N S$, Not significant. ${ }^{*}$ Controls (new cannulas), $n=5$.

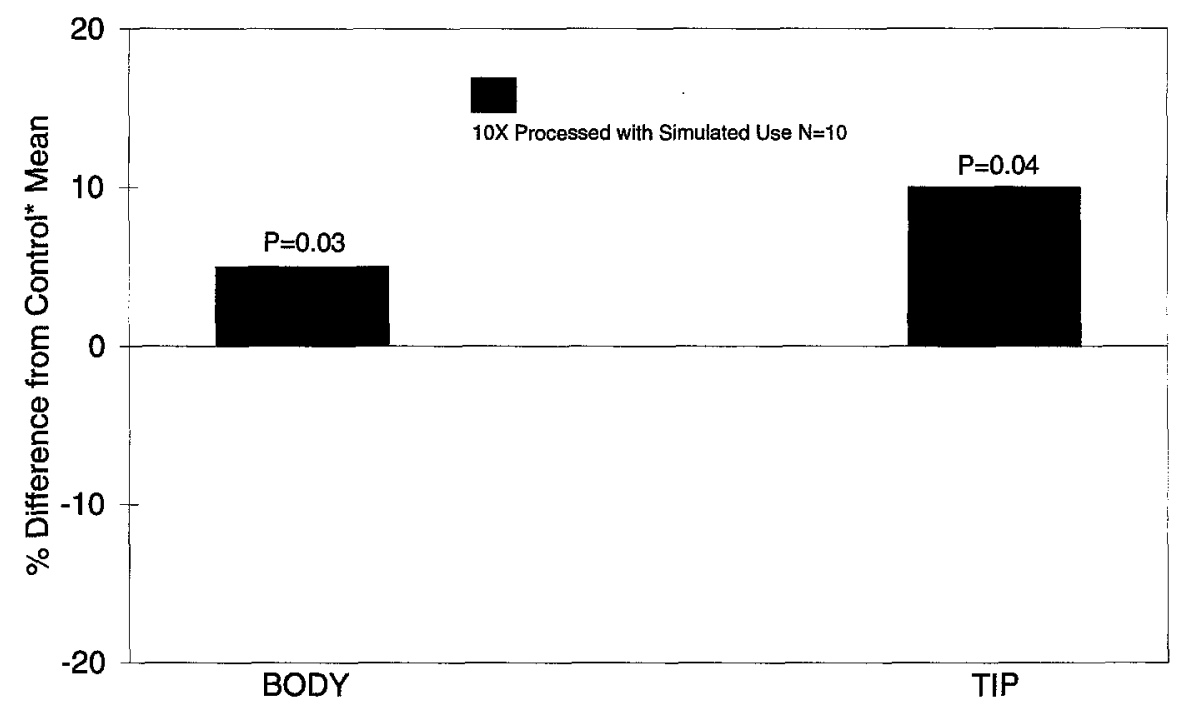

Fig. 7. Function testing-Bending stiffness, $3 \mathrm{M} / \mathrm{Sarns}$ arterial cannula $\left(22^{\circ} \mathrm{C}\right) .{ }^{*} \mathrm{Controls}$ (new cannulas), $n=6$.

found in the body of the RMI dual-stage cannulas, whereas a nonsignificant decrease in stiffness was found in the tip (Fig. 5). An increase in the body and tip-bending stiffness was found to be nonsignificant for the RMI single-stage cannulas (Fig. 6). Bending stiffness of the $3 \mathrm{M} /$ Sarns cannulas showed a statistically significant yet small increase as compared with the new (unused) cannulas for both the body and tip (Fig. 7). All mean changes in the three cannulas were $20 \%$ or less and virtually undetectable by manual manipulations. Tensile stiffness of the body of the RMI dual-stage venous cannulas increased by less than $15 \%$, a change that was statistically significant (Fig. 5). The tensile stiffness of the body of the RMI single-stage venous cannulas showed an increase of less than $10 \%$, which was not a statistically significant change. Changes in tensile stiffness and other tensile properties in the body and 


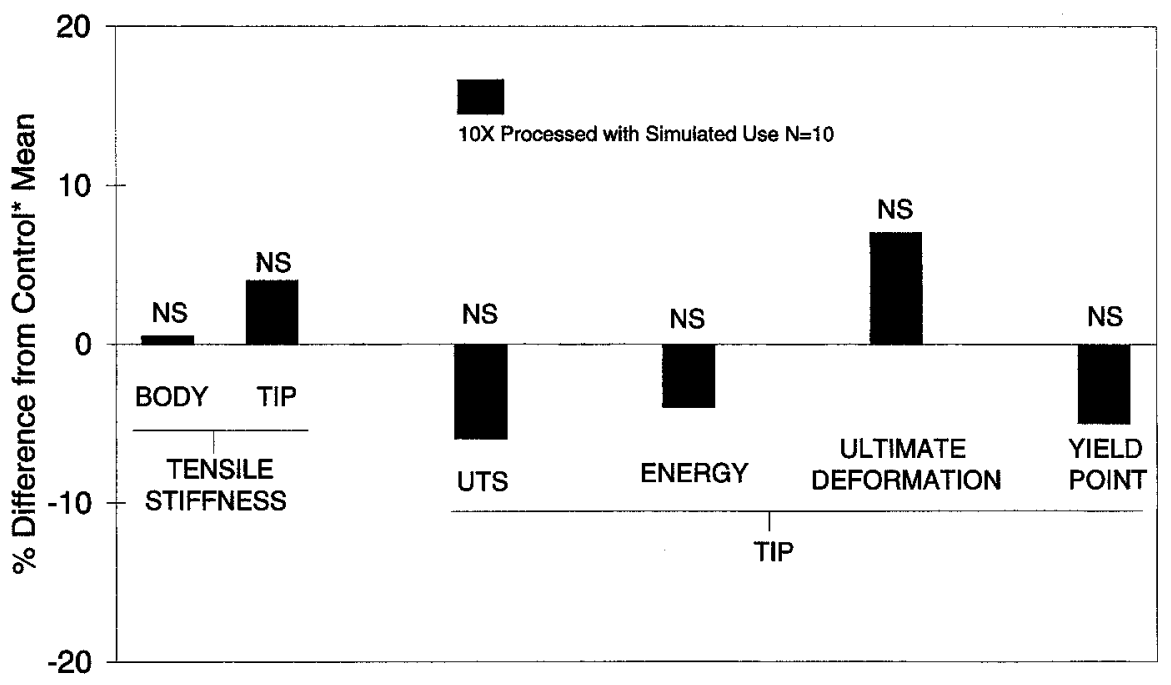

Fig. 8. Function testing-Tensile performance, $3 \mathrm{M} /$ Sarns arterial cannula $\left(22^{\circ} \mathrm{C}\right) . N S$, Not significant; UTS, Ultimate tensile strength. ${ }^{*}$ Controls (new cannulas), $n=5$.

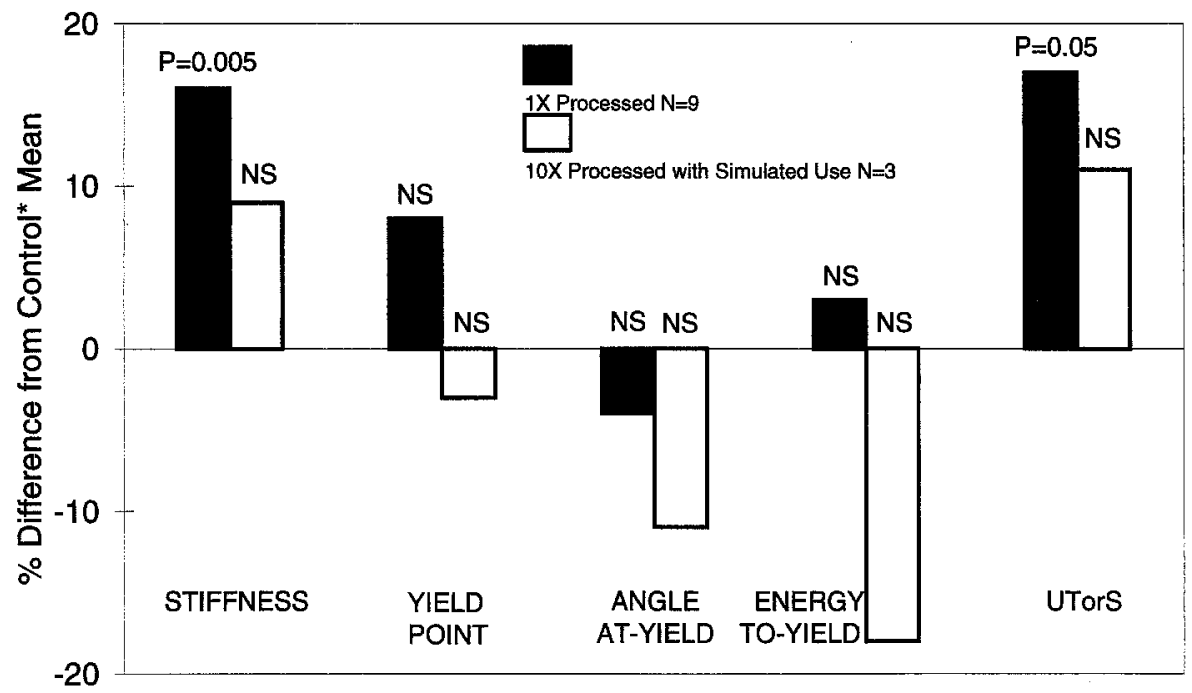

Fig. 9. Function testing-Torsion performance, 3M/Sarns arterial cannula $\left(22^{\circ} \mathrm{C}\right) . N S$, Not significant; UTorS, ultimate torsional strength; ${ }^{*}$ Controls (new cannulas), $n=9$.

tip of the reprocessed $3 \mathrm{M} / \mathrm{Sarns}$ cannulas were less than $10 \%$ and not statistically significant (Fig. 8). After reviewing the new and reprocessed cannulas with a cardiac surgeon, we deemed a change in each test parameter of less than $20 \%$ to be clinically acceptable.

Because of the unique tip design of the $3 \mathrm{M} / \mathrm{Sarns}$ arterial cannulas that incorporates struts, the torsional performance of this design was also evaluated in a mechanical shear test. As with bending stiffness and tensile properties, torsional properties showed changes of less than $20 \%$ from the controls (Fig. 9). Although statistically significant changes were noted in some cases, the changes were virtually undetectable by qualitative evaluation (manual manipulation) and, in the opinions of the consulting cardiac surgeons, were not considered clinically significant. All changes in the cannulas, after clinical or simulated use, whether statistically significant or not, were less than $20 \%$ from the 
Table II. Summary of materials and functional testing for RMI dual-stage venous perfusion cannulas at room temperature $\left(22^{\circ} \mathrm{C}\right)$

\begin{tabular}{|c|c|c|c|c|}
\hline Test type & Property & $\begin{array}{l}\text { Unused cannulas } \\
\text { (mean }[ \pm S D])\end{array}$ & $\begin{array}{c}\text { Simulated-use } \\
\text { cannulas } \\
\text { (mean }[ \pm S D I)\end{array}$ & $\begin{array}{c}p \text { Value }^{*} \\
(\% \text { change })\end{array}$ \\
\hline \multirow[t]{2}{*}{ Materials } & Appearance & Control & Scoring at proximal end & - \\
\hline & Color & Control & Slight yellowing & - \\
\hline \multirow{4}{*}{ Bending } & Tactile/texture & $\begin{array}{c}\text { Control } \\
n=5\end{array}$ & $\begin{array}{c}\text { No apparent difference } \\
n=10\end{array}$ & - \\
\hline & Bending stiffness (in) & & & \\
\hline & Body & $2.71(0.15)$ & $3.20(0.20)$ & $<0.001(-15 \%)$ \\
\hline & Tip & $0.14(0.02)$ & $0.17(0.04)$ & NS $(-21 \%)$ \\
\hline \multirow[t]{2}{*}{ Tensile } & Tensile stiffness $(\mathrm{N} / \mathrm{mm})$ & $n=5$ & $n=10$ & \\
\hline & Body & $1.84(0.14)$ & $2.07(0.11)$ & $0.004(+13 \%)$ \\
\hline
\end{tabular}

$S D$, Standard deviation; $N S$, nonsignificant $(p>0.05)$.

*Student's two-tailed unpaired $t$ test; $\alpha=0.05$.

Table III. Summary of materials and functional testing for RMI single-stage venous perfusion cannulas at room temperature $\left(22^{\circ} \mathrm{C}\right)$

\begin{tabular}{|c|c|c|c|c|}
\hline Test type & Property & $\begin{array}{l}\text { Unused cannulas } \\
\text { (mean }[ \pm S D])\end{array}$ & $\begin{array}{c}\text { Simulated-use } \\
\text { cannulas } \\
\text { (mean }[ \pm S D] \text { ) }\end{array}$ & $\begin{array}{c}p \text { Value }^{*} \\
(\% \text { change })\end{array}$ \\
\hline \multirow[t]{2}{*}{ Materials } & Appearance & Control & Scoring at proximal end & - \\
\hline & Color & Control & No apparent difference & - \\
\hline \multirow[t]{4}{*}{ Bending } & Tactile/texture & $\begin{array}{l}\text { Control } \\
n=5\end{array}$ & $\begin{array}{l}\text { No apparent difference } \\
\qquad n=10\end{array}$ & - \\
\hline & Bending stiffness (in) & & & \\
\hline & Body & $4.46(0.22)$ & $4.23(0.21)$ & $\mathrm{NS}(+9 \%)$ \\
\hline & Tip & $0.30(0.04)$ & $0.27(0.04)$ & NS $(+10 \%)$ \\
\hline \multirow[t]{2}{*}{ Tensile } & Tensile stiffness $(\mathrm{N} / \mathrm{mm})$ & $n=5$ & $n=10$ & \\
\hline & Body & $0.92(0.10)$ & $0.99(0.21)$ & $\mathrm{NS}(+8 \%)$ \\
\hline
\end{tabular}

$S D$, Standard deviation; $N S$, nonsignificant $(p>0.05)$.

${ }^{*}$ Student's two-tailed unpaired $t$ test; $\alpha=0.05$.

control (new) values. Tables II to IV compare the materials and functional test data for all three cannula models.

Because the cannulas in clinical use may be subjected to temperatures ranging from $4^{\circ}$ to $40^{\circ} \mathrm{C}$, a specialized study of the $3 \mathrm{M} / \mathrm{Sarns}$ arterial cannulas was carried out to evaluate the bending stiffness, tensile stiffness, and durometer properties at these extreme temperatures. No statistically significant differences were noted between the control (new) devices and reprocessed cannulas at $4^{\circ}$ and $40^{\circ} \mathrm{C}$ (Fig. 10).

Biocompatibility testing. Infrared analysis of the RMI dual-stage venous and 3M/Sarns arterial cannula extracts showed no important qualitative differences in extractions between unused and simulated-use cannulas. The individual peaks in the infrared scans were virtually identical and unable to be identified with the available references of the independent laboratory. These results would indi- cate that the sterilization process does not create any additional leachates from the product. The extractable residue (which was greater from the control devices) appears to come directly from the cannula and is not a result of the sterilization process. The sodium hydroxide and hydrochloric acid extracts also did not differ significantly from controls. The studies indicate no significant retention or accumulation of sterilant components in the cannulas, even after 11 sterilization cycles. Residual hydrogen peroxide and peracetic acid levels within the sterilization processor after sterilization were no more than $5 \mathrm{ppm}$ for peracetic acid and no more than $0.5 \mathrm{ppm}$ for peroxide. These low levels indicate that the sterilant was adequately removed from the processor by the sterile water rinses.

The cytotoxicity test is considered to be highly sensitive and, in most cases, is more sensitive than the muscle implantation test. ${ }^{4}$ The reprocessed RMI 
Table IV. Summary of materials and functional testing for 3M/Sarns arterial perfusion cannulas at room temperature $\left(22^{\circ} \mathrm{C}\right)$

\begin{tabular}{|c|c|c|c|c|}
\hline Test type & Property & $\begin{array}{l}\text { Unused cannulas } \\
\text { (mean }[ \pm S D] \text { ) }\end{array}$ & $\begin{array}{c}\text { Simulated-use } \\
\text { cannulas } \\
\text { (mean }[ \pm S D] \text { ) }\end{array}$ & $\begin{array}{l}p \text { Value* } \\
(\% \text { change })\end{array}$ \\
\hline \multirow[t]{6}{*}{ Materials } & Appearance & Control & No apparent difference & - \\
\hline & Color & Control & Slight yellowing & - \\
\hline & Tactile/texture & $\begin{array}{c}\text { Control } \\
n=6\end{array}$ & $\begin{array}{l}\text { No apparent difference } \\
\qquad n=5\end{array}$ & - \\
\hline & Hardness & & & \\
\hline & Wire-reinforced body & $68.0(4.3)$ & $70.6(2.6)$ & NS $(+4 \%)$ \\
\hline & Nonreinforced body & $51.8(2.5)$ & $50.6(5.3)$ & $\mathrm{NS}(-2 \%)$ \\
\hline \multirow[t]{4}{*}{ Bending } & & $n=6$ & $n=10$ & \\
\hline & Bending stiffness (in) & & & \\
\hline & Body & $3.22(0.08)$ & $3.04(0.17)$ & $0.03(+5 \%)$ \\
\hline & Tip & $0.86(0.06)$ & $0.80(0.05)$ & $0.04(+10 \%)$ \\
\hline \multirow[t]{8}{*}{ Tensile } & & $n=5$ & $n=10$ & \\
\hline & Tensile stiffness $(\mathrm{N} / \mathrm{mm})$ & & & \\
\hline & Body & $1.79(0.12)$ & $1.80(0.21)$ & $\mathrm{NS}(t<1 \%)$ \\
\hline & Tip & $1005.9(188.8)$ & $1043.5(82.3)$ & $\mathrm{NS}(+4 \%)$ \\
\hline & Tensile yield strength $(\mathrm{N})$ & $219.0(28.4)$ & $207.1(17.9)$ & $\mathrm{NS}(-5 \%)$ \\
\hline & Ultimate tensile strength $(\mathrm{N})$ & $262.8(24.1)$ & $245.3(11.9)$ & $\operatorname{NS}(-6 \%)$ \\
\hline & Elongation-at-break (mm) & $0.90(0.07)$ & $0.96(0.05)$ & $\mathrm{NS}(+7 \%)$ \\
\hline & Energy-to-break (mJ) & $179.3(8.6)$ & $172.2(15.2)$ & NS $(-4 \%)$ \\
\hline \multirow[t]{6}{*}{ Shear (tip only) } & & $n=9$ & $n=3$ & \\
\hline & Torsional stiffness (N-mm/deg) & $37.1(3.5)$ & $40.4(4.1)$ & $\mathrm{NS}(+9 \%)$ \\
\hline & Torsional yield strength (N-mm) & $170.5(33.7)$ & $165.1(18.2)$ & $\mathrm{NS}(-3 \%)$ \\
\hline & Ult. torsional strength (N-mm) & $268.4(43.1)$ & $298.9(57.6)$ & $\mathrm{NS}(+11 \%)$ \\
\hline & Angle-at-yield (deg) & $5.60(1.00)$ & $4.97(1.24)$ & NS $(-11 \%)$ \\
\hline & Energy-to-yield (mJ-deg) & $590.6(275.9)$ & $480.8(227.6)$ & NS $(-18 \%)$ \\
\hline
\end{tabular}

$S D$, Standard deviation; $N S$, nonsignificant $(p>0.05)$.

*Student's two-tailed unpaired $t$ test; $\alpha=0.05$.

dual-stage venous and $3 \mathrm{M} /$ Sarns arterial cannulas did not meet the USP requirements for in vitro cytotoxicity. However, the USP (in vivo) muscle implantation tests showed no significant macroscopic reactions to the test materials when compared with a control implant (USP negative material). Microscopic examination of the implants resulted in a material rating of nonirritant to slight irritant. Therefore, although this discrepancy in the results exists, the positive cytotoxicity test results can generally be dismissed if the balance of in vivo testing proves acceptable.

In vitro hemolysis testing on the two cannula models showed that hemolysis was below $0.5 \%$; thus the cannulas were considered to be nonhemolytic. Bacterial endotoxins in the extracts were less than $0.125 \mathrm{EU} / \mathrm{ml}$ (endotoxin units per milliliter) for the RMI dual-stage cannulas and less than $0.5 \mathrm{EU} / \mathrm{ml}$ for the $3 \mathrm{M} / \mathrm{Sarns}$ arterial cannulas. Hence the processed cannulas met the requirements of the FDA and USP for medical devices $(\leq 0.5 \mathrm{EU} / \mathrm{ml})$ and are considered nonpyrogenic. Last, systemic toxicity and intracutaneous toxicity studies showed that the extracts were nonirritants. Therefore the cannulas met USP requirements and are considered nontoxic.

In vivo testing. Cardiac valve replacement experiments in sheep using the RMI single-stage venous perfusion cannulas showed no differences in device use or performance (insertion, handling, function, and removal) between the new and reprocessed (clinically used once and processed four times without simulated use) cannulas. In these studies, the coronary bypass times ranged from 71 to $159 \mathrm{~min}$ utes, pump temperatures ranged from $30^{\circ}$ to $40^{\circ} \mathrm{C}$, and heparin infusion during bypass ranged from 11,000 units to 17,000 units. No blood component deposits or surface clots were detected on the cannulas. In addition, thromboembolism did not occur in the pulmonary arteries, and the infection rate in the test animals was the same as that in control animals. Infection, which was determined by a temperature rise of more than $40^{\circ} \mathrm{C}$ with a normal white blood cell count, was noted in one test case and in one control case. Evaluation by the cardiac 


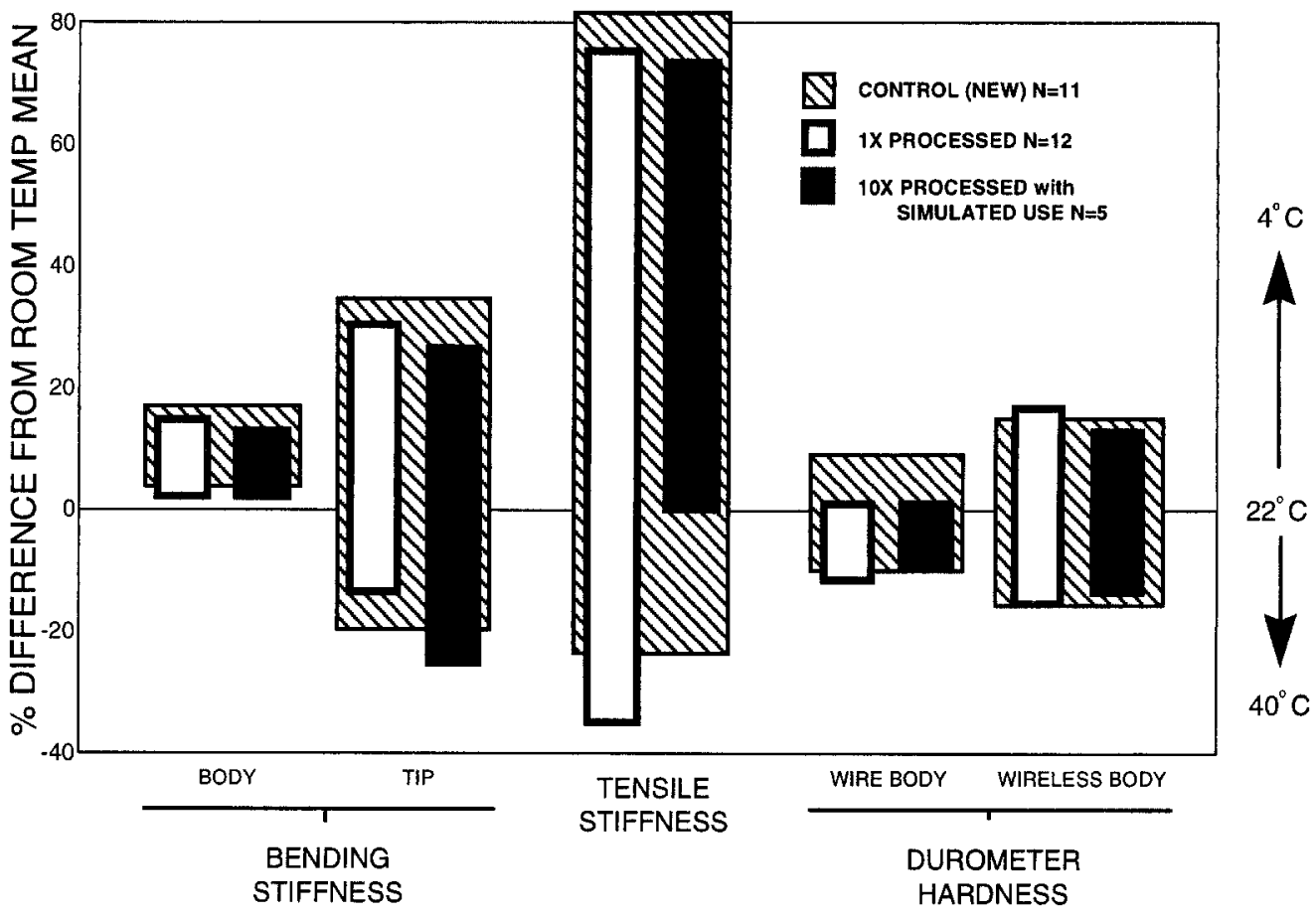

Fig. 10. Function testing-Temperature sensitivity, 3M/Sarns arterial cannula.

surgeon showed that performance of the reprocessed cannulas is comparable with that of new ones.

Cost minimization analysis. A basic cost minimization analysis was undertaken to determine whether it would be economically feasible to use perfusion cannulas five times (once when new and four additional times with required reprocessing), assuming that safety and efficacy after each use remained equal to that of new cannulas. Total and incremental costs of reusing these perfusion cannulas four times after their initial use were calculated. To provide a complete cannula set per surgical case (including multiple sizes), the STERIS General Processing Container can be modified to accommodate five cannulas. It is assumed that all cannulas can be reprocessed four times and that five cannulas can be reprocessed during a single sterilization cycle.

Costs for a single sterilization cycle include the sterilant (\$5), consumable supplies (\$2), and capital equipment (\$3). Capital costs are based on an initial cost of $\$ 16,200$ for a STERIS SYSTEM 1 amortized over 5 years (assuming that the system would provide 1000 cycles each year). The cost for a single sterilization cycle would, therefore, be $\$ 10$. When processing five cannulas per cycle, the cost of ster- ilizing a single cannula would be $\$ 2$ (all cost estimates are from STERIS Corporation). To this figure must be added a reprocessing cost (\$1.77), which is based on labor costs of $\$ 10$ per hour (data provided by The Cleveland Clinic Foundation). Therefore the incremental cost of reusing a cannula equals $\$ 3.77$. In contrast, the cost of a new cannula is $\$ 20$ (sources: Research Medical Inc. and $3 \mathrm{M} /$ Sarns Corporation).

The annual total and incremental costs per procedure for using new and reused cannulas are provided in Table V. By reusing cannulas, the incremental cost per procedure is $\$ 18.64$, which is a $65 \%$ savings over the current cost of $\$ 53.13$. If implemented, a program to reuse perfusion cannulas would save the institution $\$ 110,364$ each year.

\section{Discussion}

Many of the currently disposable medical devices now being considered for reuse (i.e., hemodialyzers, perfusion cannulas) were originally designed for reuse. With the increasing availability of mass production manufacturing techniques and less expensive materials, these devices were made and sold as disposables. The durability of many disposable products, particularly high-cost and/or high-volume 
products, has prompted studies into the safety and efficacy of their reuse.

Several studies have attempted to address the many issues surrounding the reuse of disposable devices. In a 1993 study on the reuse of perfusion cannulas, ${ }^{5}$ cannulas were rinsed free of blood, processed in a washer-sterilizer at $140^{\circ} \mathrm{C}$ for $30 \mathrm{~min}$ utes, evaluated for breaks and defects, wrapped, sterilized in ethylene oxide for 3 hours, and aerated for 12 hours before clinical use. This study contained no discussion of safety and efficacy of the reprocessing methods, although recycling of cannulas had been used as a method of cost savings for many years. Other studies of disposable cardiovascular catheters reused for catheterization and angioplasty generally reported clinical outcomes only, ${ }^{6-8}$ although, in some studies, safety and functional evaluations on devices have been carried out to a limited degree. ${ }^{9,10}$

In contrast to past studies, the current study presents a comprehensive approach to evaluating reuse of disposable medical devices. Perfusion cannulas were selected for investigation and used to create a model reprocessing program because these cannulas were at one time routinely reprocessed in the United States and because they have a simple design. The following testing was performed on the perfusion cannulas most frequently used in the institution: sterilization efficacy, device materials and function assessments, biocompatibility assessments, and an animal study. The liquid chemical sterilization system used in this study was chosen for its ability to sterilize temperature-sensitive products and provide rapid turnaround times (about 30 minutes after cleaning and decontamination).

With bioburden levels higher than those expected in clinical use, and for an inoculum of Bacillus subtilis spores, an organism highly resistant to sterilization, cannula sterility after reprocessing was achieved in every case. The level of recoverable bioburden on the cannulas was noted to be in the range of $10^{3}$ to $10^{4} \mathrm{cfu}$ per device. This level of recoverable bioburden does not meet the current American Society for Testing and Materials requirement of $10^{6} \mathrm{cfu}$ per device, which was established after the cannula sterilization testing was completed. ${ }^{11}$ In a separate study using the same sterilization system, RMI dual-stage venous return cannulas were soiled with a Bacillus subtilis $\left(10^{7} \mathrm{cfu} / \mathrm{ml}\right)$ spore suspension consisting of $5 \%$ bovine whole blood in physiologic saline solution $(0.9 \% \mathrm{NaCl}){ }^{12}$
Table V. Cost minimization analysis comparing the annual costs of new perfusion cannulas to the costs of perfusion cannulas used five times*

\begin{tabular}{lccc}
\hline & $\begin{array}{c}\text { Without } \\
\text { reuse }\end{array}$ & $\begin{array}{c}\text { With } \\
\text { reuse }\end{array}$ & $\begin{array}{c}\text { Savings } \\
\text { with cannula } \\
\text { reuse }\end{array}$ \\
\hline Number bought/year & 8500 & 1700 & 6800 \\
Cost of first use & $\$ 20.00$ & $\$ 20.00$ & \\
Cost of second use & - & $\$ 3.77 \dagger$ & \\
Cost of third use & - & $\$ 3.77$ & \\
Cost of fourth use & - & $\$ 3.77$ & \\
Cost of fifth use & - & $\$ 3.77$ & \\
Total cost/year & $\$ 170,000$ & $\$ 59,636$ & $\$ 110,364$ \\
Number of procedures/year & 3,200 & 3,200 & \\
Incremental cost/procedure & $\$ 53.13$ & $\$ 18.64$ & $\$ 34.49$ \\
\hline
\end{tabular}

*Initial use (when new) and four additional reuses (each involving resterilization).

$\doteqdot$ See text for derivation of unit cost of sterilization.

The devices were inoculated by being totally submerged in the spore suspension and then air dried for at least 30 minutes. The level of inoculation recovered on the devices ranged from $0.98 \times 10^{6}$ to $2.5 \times 10^{6} \mathrm{cfu}$ or more per device. There was no cleaning or decontamination of the devices after inoculation and before the sterilization cycle. After each cycle, all test sites ( $n=60$; three cannulas, four sites per cannula, five process cycles per cannula) showed the absence of the test organism, which indicates that soiling with a high level of inoculum containing whole blood and salt has no effect on the ability of the system to sterilize the cannulas. One reason for the large discrepancy in the bioburden recoveries between the two studies may be that different inoculation techniques were used. In this study, the device was inoculated with the use of a syringe by partially wetting the cannula surfaces with the spore suspension, whereas in the blood/salt study the device was completely immersed in the inoculum.

The cannulas showed no physical or mechanical changes that would appear to affect their use or function. Where defects were noted, or where scoring occurred, visual inspection by trained staff would be sufficient to eliminate these cannulas from further use.

Analysis of cannula sterilant extracts found no significant differences between new and reprocessed cannulas. The positive in vitro cytotoxicity results may only reflect the high sensitivity of this test method. ${ }^{4}$ The fact that the cannulas passed the other biocompatibility tests, including the muscle 
implantation test, and that no adverse reactions were observed in the animal model (one of the cannula models evaluated), showed that the reprocessed cannulas are safe.

The results of this testing suggest that a clinical trial be designed and carried out to assess the clinical utility and overall economic impact of reuse of perfusion cannulas. As reuse is expanded to other products after careful laboratory-based research and clinical trials, the savings in total health care costs should be substantial. With the reduction of health care reimbursement, removing costs from the system is the only way in which institutions can continue to provide the highest quality medical care to all patients. The successful reuse of traditionally disposable medical products and devices must be associated with the highest quality of laboratory-based research and a willingness of regulatory bodies to review the scientific data objectively and rigorously and, if positive, to approve clinical trials.

The statistical analysis was performed by Michelle Secic, MS. We thank Tom Lang for editorial assistance in the preparation of this manuscript.

\section{Note}

This feasibility study was undertaken to determine whether a more substantial study of resterilization of disposable devices would be reasonable to pursue. The study was not intended to demonstrate the safety and effectiveness of reusing any specific device or resterilizing with the sterilization process in the study. Neither the devices tested nor the sterilization process used to resterilize them are cleared by the U.S. Food and Drug Administration for reuse or resterilization of devices labeled for single use.

\section{REFERENCES}

1. Apelgren KW, Blank ML, Slomski CA, Hadjis NS. Reusable instruments are more cost-effective than disposable instruments for laparoscopic cholecystectomy. Surg Endosc 1994; 8:32-4.

2. American Society for Testing and Materials. Standard Test Method for Apparent Bending Modulus of Plastics by Means of a Cantilever Beam, D747-90. Philadelphia: American Society for Testing and Materials; 1990.

3. American Society for Testing and Materials. Standard Method of Test for Tensile Properties of Plastics, D638-72. Philadelphia: American Society for Testing and Materials; 1972.

4. Wallin RF. Scoring biological safety tests: What's a pass or a fail? Med Plast Biomater 1994;1:38-41,55.

5. Novick RJ. Recycling of cardiopulmonary bypass cannulae: a risk-benefit analysis. Can J Surg 1993;36:431-4.

6. Jacobson JA, Schwartz CE, Marshall HW, Conti M, Burke JP. Fever, chills, and hypotension following cardiac catheterization with single- and multiple-use disposable catheters. Cathet Cardiovasc Diagn 1983;9:39-46.

7. Frank U, Herz L, Daschner FD. Infection risk of cardiac catheterization and arterial angiography with single- and multiple-use disposable catheter. Clin Cardiol 1988;11:785-7.

8. Plante S, Strauss BH, Goulet G, Watson R, Chisholm RJ. Reuse of balloon catheters for coronary angioplasty: A potential cost-saving strategy? J Am Coll Cardiol 1994;24:1475-81.

9. Conseil d'évaluation des technologies de la santé du Québec. The reuse of single-use cardiac catheters: safety, economical, ethical and legal issues. Can J Cardiol 1994;10:413-21.

10. Mussivand T, Mackenzie AMR, Blohon R, Duguay D, Rajagopalan K, Holmes K. Cardiac catheter reuse pilot study [abstract]. ASAIO J 1995;41:92.

11. American Society for Testing and Materials. Standard Test Method for Determination of Effectiveness of Sterilization Processes for Reusable Medical Devices, E1766-95. Philadelphia: American Society for Testing and Materials; 1995.

12. Bucci DM, Richardson D, Bloom D, Malchesky PS. Effect of blood soiling on sterilization efficacy. Presented at the Thirty-first Annual Meeting of Association for Advancement of Medical Instrumentation, 1996, Philadelphia; June 2, 1996. 